\title{
Link between BIM and energy simulation
}

\author{
M. Senave ${ }^{1} \&$ S. Boeykens ${ }^{2}$ \\ ${ }^{1}$ Building Physics Section, KU Leuven, Belgium \\ ${ }^{2}$ Department of Architecture, KU Leuven, Belgium
}

\begin{abstract}
The emerging policies on building energy performance, developed by governments to face growing environmental concerns, stress the need for means to predict the future performance of a scheme in order to ensure that the as-built project will meet regulations. Several methods may enable us to expeditiously make the link between a Building Information Model (BIM) and energy simulation tools during the design process. We can differentiate between energy evaluations accomplished within the BIM software and those requiring data transfer from the model to specific analysis tools. Likewise, we can make a distinction between evaluations applicable during the concept and the design phase. The intention of the research described in this paper was to gain insight into the technical abilities and to point out issues concerning the relation between BIM and energy simulation in the different stages of design, with an emphasis on the conceptual stage. Hence a concise overview of the appropriate literature has been made, followed by five case studies. We get acquainted with an arsenal of tools, some of which still in a scientific stage, and see all kinds of promising developments on the market, both from BIM applications and from energy simulation tools. Software used includes ArchiCAD, Revit, Sefaira, the Space Boundary Tool, EnergyPlus, SketchUp, Open Studio and the EPB software imposed by the Belgian government.
\end{abstract}

Keywords: BIM, building energy performance (BEP), energy simulation (software), interoperability, information query, data transfer, design stages

\section{Introduction}

Latterly, Building Information Modelling (BIM) has proven to be one of the major innovations in the building industry. Being more than just a software tool that improves the efficiency of design work, BIM is profoundly changing the 
construction process and the way in which the partners of a building team collaborate (Eastman et al. [1]). Around the world an increasing adoption by architects and building companies can be observed (Bernstein and Jones [2], ArchVision [3]).

Furthermore, we are faced with growing environmental issues, such as the inevitable depletion of fossil energy reserves and the greenhouse effect. Under growing scrutiny from the public opinion, governments impose restrictions on the energy consumption of buildings. This enlarges the need for means to predict the energy performance of building projects and to ensure they will meet regulations.

Building energy performance (BEP) tools are generally divided into two categories according to the purpose of the calculation: design tools and simulation tools (Maile et al. [4]). Design tools take worst-case scenarios into account to determine the optimal size of HVAC systems. Energy simulation tools often include dynamic calculations that consider a whole year. They can be used to assess the energy demand, indoor environmental quality, carbon dioxide emission, pay-back periods of energy saving measures, and so on. The results of these calculations are a valuable source of information, not only to compare alternative solutions in the conceptual phase or to check throughout the design process if the energy performance is on track to meet regulations, but also for Model Predictive Control in the operational phase. Although many cases are generally valid for a link between BIM and BEP software, the focus in this research was put on energy simulation tools.

The input data needed to endorse an assessment of the BEP include information on the building envelope and thermal mass, weather data, information on the building services, internal loads, operating strategies and schedules and simulation specific parameters. At the start of a project the design team will focus on the building and assume default systems with fixed efficiencies. In the later stages of design rather fixed building parameters are assumed and emphasis is put on the building services. Accurate input data are a prerequisite for the generation of reliable simulation results. Furthermore, simulation software capable of using these data to evaluate a particular design decision, analysis of the results that focuses on the design decision and a competent user are required (Bazjanac et al. [5]).

During the design process, the performance of a scheme might be optimized by a sequence of analysis and feedback. BIM consolidates this iteration, since the intelligent model serves as a common, continuously up-to-date database that is steadily refined until the desired objectives are met (fig. 1). To this purpose proper methods of deriving and converting the input for the simulation model are essential.

The ideal and automated integration of multi-criteria design evaluation with BIM is not fully implemented yet. Instead of automatically generating a Building Energy Model (BEM model), manually re-entering of architectural and technical information into static calculation or dynamic simulation software is common practice: a highly inefficient working method, prone to errors. Moreover, Bazjanac and Kiviniemi [6] states that a virtual model for energy assessment entails 
DESIGN PROCESS

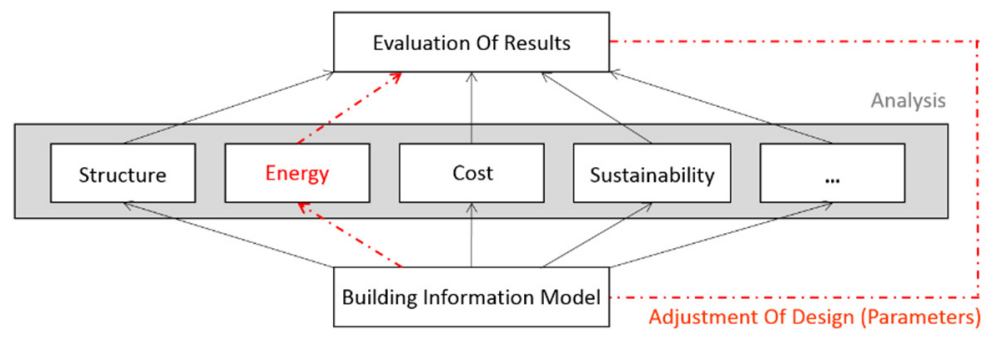

Figure 1: BIM facilitates an iterative workflow. In this paper we will merely focus on the aspect 'energy performance'. Because of the mutual influence of structure, cost, sustainability, etc. multi-criteria design evaluation is, however, preferable.

divergent properties than one created from an architectural point of view. Hence the data embedded in the BIM has to undergo a threefold transformation: reduction, translation and interpretation. Such operations are time-consuming and complex. Due to misinterpretations later reproductions might even be impossible. Therefore, the transformation process should be automated and standardised.

The generation of an appropriate energy model and the subsequent evaluation can be accomplished within BIM software. We categorise this integrated type of energy assessments as 'internal'. By contrast, 'external' energy evaluations require data transfer from the model to specific analysis tools.

BIM provides the building team with a substantial amount of information in an early stage, so the enhancement of the performance of a scheme can be considered at a time when the cost of changes is relatively low. Yet, even if the amount of information available on the elements and objects (the Level of Detail) is high in the conceptual stage, this does not necessarily mean that all these characteristics have been analysed thoroughly and have been agreed upon, which is necessary to obtain a high Level of Development (LOD). This might make the results of the simulations in the conceptual and preliminary stages to exhibit a low reliability and large dispersion.

The authors of this paper want to explore the technical viability and point out issues concerning the link between BIM and energy simulation, emphasizing on the conceptual stage. Primarily we contemplate the necessary data transfer between the model and the energy simulation program. A building information model usually is abounding in data which makes it difficult to be used straight away. Which information stored in the model must minimally be handed over in order to meet the needs of the energy simulation? How can we extract these data from the model and introduce them into the analysis software? Secondly we discuss the assimilation of BIM and the energy performance analysis into the design process. Can a virtual building easily be evaluated against governmental regulations and can one readily see the consequences of the adjustment of a parameter on the energy consumption? Finally we consider the relation between BIM software packages, increasingly providing the user with built-in modules for 
energy evaluation, and BEP simulation software with improving graphical input and visualisation of results.

Five case studies, selected on the basis of two criteria, were accomplished. Firstly we differentiate between energy evaluations performed by tools external to BIM software (case 1, 2 and 4) and those integrated within BIM (case 3 and 5). The second criterion relates to the design phase in which the model is analysed: evaluations made in the concept phase (case 4 and 5) or during the design stage (case 1,2,3).

Most case studies are implemented on a detached house comprising two floors and a flat roof. To ensure that the findings are not of limited validity, two variants have been formulated: a house with a pitched roof and a terraced house.

\section{Findings of the case studies}

\subsection{Case Study 1: external/design stage - energy performance evaluation according to the Belgian EPB regulation}

The objective of this case study is to investigate if a BIM model containing building elements of a higher level of development facilitates the calculation of the BEP according to the EPB Directive that applies in Belgium. Both for new buildings and renovations it is mandatory to use the 'EPB software tool' to assess the BEP, so authorities may verify compliance with the regulatory demands on the degree of insulation and the use of primary energy. The program's file format is not open, and there is no possibility to import CAD or BIM files. So the requisite data on geometry, thermal properties of materials, building services, etc. should be collected from the BIM model and entered manually in the EPB program.

BIM software packages offer several possibilities for extracting model data. To obtain information embedded in a Graphisoft ArchiCAD 17 model, one can for instance generate 'lists' or 'schedules', create an IFC file or use the 'element information' tool. In this case study these data extraction methods are specifically used to collect the necessary information for the EPB software. Two examples will be cited.

EPB has certain rules for how the geometry of a building should be entered. One of the principles is that the outer dimensions should be taken into account (Vlaams Energieagentschap [7]). Extraction methods that can comply with these rules had to be invented. As ArchiCAD's integrated Energy Evaluation tool utilises net room volumes, it was not directly applicable and several separate ArchiCAD Zones and Morphs (freeform 3D volumes) had to be modelled just to derive the required geometric data (protected volume, heat loss area, gross floor area). This proved to be an intensely complex and inefficient task.

To determine the area of the window profiles, one could simply rely on the Energy Evaluation tool, because it automatically provides this information. The tool however uses another measurement method than the one prescribed by EPB: it identifies the integral profile area, instead of solely the part between the reveals. Consequently a 'schedule' with the appropriate parameter as schedule field could be used to list the correct window profile area. 
The various analyses showed there is no single, fully automatic, adequate method obtainable. At all times an amalgam of methods should be applied to acquire the desired data. Part of the research findings have been summarised in table 1, which indicates if the data needed for the EPB assessment could be extracted from the BIM model in one way or another. For the extensive table we refer to (Senave [8]). Alas, only a subset of the required information could be derived directly from the virtual model. In a number of cases the information was not readily available and had to be obtained through additional, time-consuming and sometimes far-fetched modelling or the creation of new elements. In other cases, the necessary information was not present. It could be a solution to attach this specific information to the elements in the BIM model.

Table 1: Part of the research findings of Case Study 1; data query for the EPB program.

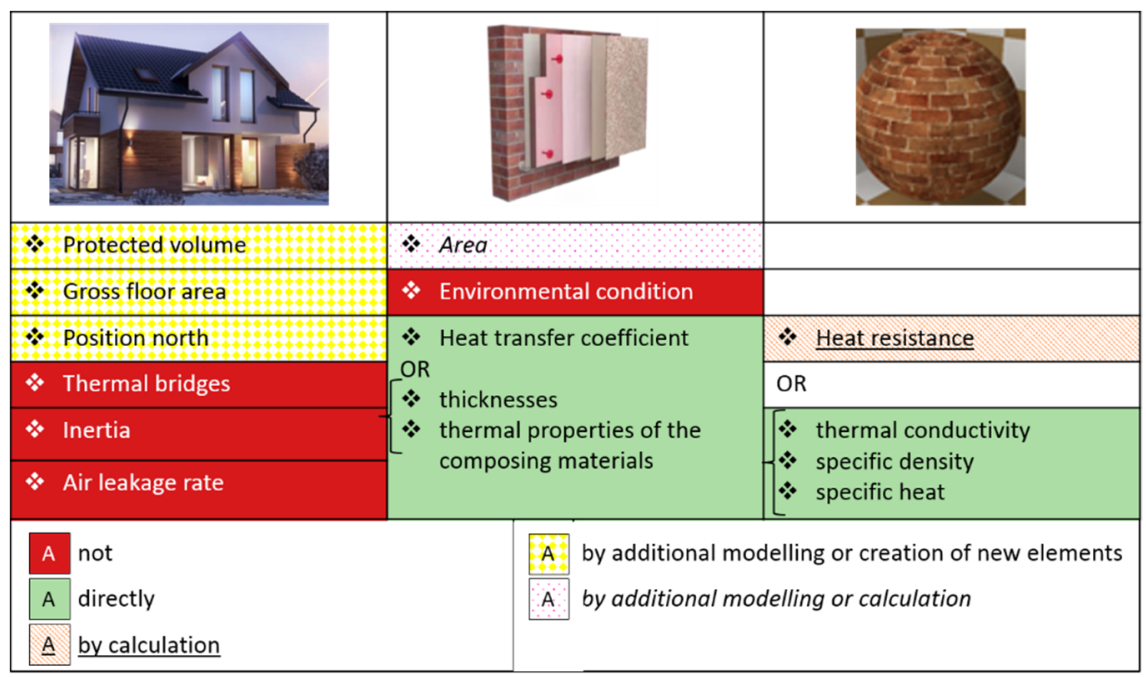

This would fit the BIM philosophy (storing all information centrally and accessible to all building partners) and make the model more of use for EPB declarations afterwards, but appears in some cases to reach the limitations of the available empty fields. Moreover, the form in which the data are available is not always as desired, making additional operations indispensable. The difference between the measurement methods available in the BIM software and those prescribed by EPB plays a crucial role in this. Specific to ArchiCAD, the weak link between the data disclosed by the Energy Evaluation tool in its Excel report and the elements in the model (e.g. the lack of ID's) complicates the process.

Through a number of stop-gap measures, it is possible to export the majority of the geometrical properties from the ArchiCAD model. The available data of the technical installations could not be recovered. In spite of all data extraction methods BIM software like ArchiCAD offers, this case study showed that a lot of difficulties arise when a user tries to obtain specific data for an external energy 
analysis. We therefore may conclude that this link and particular workflow, even if technically feasible, certainly is not user-friendly and hence has little chance to succeed.

\subsection{Case Study 2: external/design stage - from BIM to an external Building Energy Simulation Program}

In a second case study we investigate if the link between a BIM model and an energy simulation package via the IFC standard is functioning properly. We therefore examine the Space Boundary Tool (SBT), developed by LBNL (SBT [9]). In this tool, meant to enable the transfer of BIM model data to EnergyPlus (fig. 2), the principles of data transformation (see $\$ 1$ ) have been implemented in a clear and demonstrative way. We put the tool to a test by means of two simple 'box buildings', developed within Graphisoft ArchiCAD 17 and Autodesk Revit 2014. When intending to perform energy simulations later on, one should keep in mind some rules while constructing the BIM model. Besides general conventions of energy models (Maile et al. [10]), the SBT and EnergyPlus also have their own requirements and limitations (O'Donnell et al. [11]).

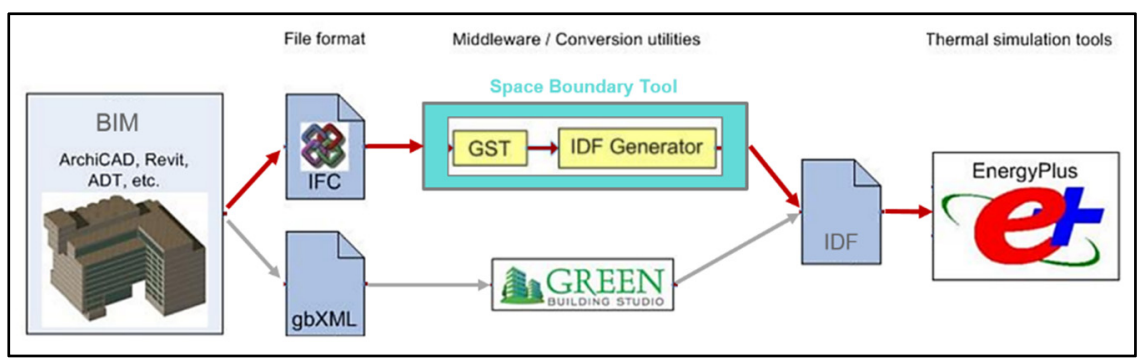

Figure 2: The SBT, a middleware tool providing the link between BIM and EnergyPlus through the IFC format (adapted from Maile et al. $[4$, p. 33]).

A BIM model saved as an IFC with a certain Model View Definition can be imported in the SBT, where the file is enriched with space boundaries. As for the IFC model developed in Autodesk Revit, the SBT seamlessly recovered all space boundaries. In the case of the ArchiCAD 17-IFC, the authors experienced some problems concerning the definition of the windows. The content of the enriched IFC file should thus be inspected.

The conversion of a BIM model into a model useful for energy evaluation through an IFC file was a complex process because in addition to the welldocumented automatic operations (Bazjanac $[12,13]$ ) a number of manual actions had to be carried out. One step in the conversion process in which human intervention is needed is the matching of the materials found in the IFC-based BIM to corresponding materials in the EnergyPlus material library. For instance, each layer of an ArchiCAD exterior wall construction has to be translated into a material layer of EnergyPlus to constitute an equivalent wall. 
The SBT only retrieves data concerning the geometry, materials and constructions from a BIM model. Consequently it asks the user to manually specify simulation and load characteristics and generates an IDF file. The geometry present in this file can be inspected visually by opening it in SketchUp (using the Open Studio Plug-in) or by requesting EnergyPlus to create a DXF output file following the analysis. The user can add information on technical systems, user behaviour and internal gains to the EnergyPlus input file later on.

A number of operations have to be executed to transfer the information embedded in a BIM model into a BEM model in order to run one simulation. Next, the BEM model can be developed further, it is, however, not possible to coordinate these adjustments to the BIM model. The other way around, the BIM model may be modified as the design evolves. But since it is not possible to automatically synchronise the two models, one has to start from scratch again with the BIM to BEM conversion.

Meanwhile, the developers of the SBT created a new, fully comprehensive tool, 'Simergy', which furthermore enables to include information on internal loads and HVAC systems in the simulation input file (Simergy [14]). This might make the SBT redundant, but it remains an instructive demonstration of the data transformation methodology.

\subsection{Case Study 3: internal/design stage - energy evaluation integrated within BIM software}

In an ideal world a variety of simulation and BIM software exists and the user can link any of them through the IFC file format. However, this seems not straightforward. Besides, BIM developers increasingly incorporate simulation tools into their modelling packages, directing to a number of advantages, the main one being the ease of working with only one software program. Graphisoft, for instance, provided its software ArchiCAD 17 with the Energy Evaluation tool which we will evaluate in this case study.

Abiding by some guidelines while modelling in BIM will advance the generation of the energy model and will provide more profound simulation prospects. These aspects concern e.g. the way the building envelope is modelled or user zones are created. Once the desired architectural concept has been developed in BIM, the Energy Evaluation tool automatically creates a corresponding Building Energy Model. The hereby generated space boundaries are listed as 'Structures' and 'Openings', along with their unique geometrical and physical properties, orientation and position relative to the thermal block to which they belong. Apart from the automatically generated BEM geometry, that may be checked visually, the accuracy of the non-visual properties of the space boundaries should be verified. A distinction must be made between data taken directly from the ArchiCAD model and which cannot be adapted (ArchiCAD model types), and other parameters (Additional data types) for which Energy Model Review suggests a rewritable value that should be inspected closely. We notice that Energy Evaluation can increasingly capture all aspects of an energy simulation: in addition to the building data, data on simulation parameters, operation profiles and heat gains, climate and environmental conditions and HVAC installations can be edited 
and assigned to thermal blocks. These are simplified data and there is a limited number of parameters, but that should be sufficient for the intended purpose: determining the primary energy consumption, $\mathrm{CO}_{2}$ emission and running costs. The output files present the results of the energy simulation in a clear, graphical way. They can be studied to check whether the results correspond to expectations, previous findings and similar projects. Caution and reserve with respect to the outcomes is appropriate, as these are in accordance with the data processing and the assumptions made by the software package. Where necessary, the model or simulation assumptions should be adjusted whereupon the procedure must be repeated. A well-thought-out composition of the architectural model from the start and a consistent modelling method afterwards remain important in order not to introduce errors when making adjustments.

\subsection{Case Study 4: external/concept stage - tools for energy analysis in the early design stages and their link with BIM}

Given the benefits of an early design evaluation, software companies have developed tools able to expeditiously assess the BEP based on a limited amount of data. In this case study we investigate the value of these tools and how they could be integrated in the BIM methodology. Successively, we will take a look at the software of Sefaira, and examine the link between SketchUp and EnergyPlus via Open Studio.

The developers of Sefaira focus on performance-based design during the concept and preliminary design stages. The plug-in analyses a design modelled in Trimble SketchUp or Autodesk Revit. Non-geometric design properties, such as the performance of the construction components and HVAC systems, as well as the internal gains, can be configured by the user. It is noteworthy that this evaluation is based on value judgment rather than numerical values, which is consistent with the reality in the conceptual design stage: one has a notion of the level of performance to be achieved, but is not yet able to designate appropriate materials or thermal values to it. The building performance is monitored and analysed in real time as the design evolves. Fast and intuitive feedback on the energy use intensity, the percentage of floor area receiving a certain level of daylight and the specific heat gains and losses is given in the 'dashboard' of the plug-in. This enables the designer to automatically see the consequences of modifications being carried out and to evaluate which building elements energetically contribute most, so as to take mitigating measures. By adjusting the model and setting parameters in straightforward way, different design options can instantly be explored and a strategy can be developed to achieve the desired results.

In the design phase, another tool of Sefaira, the 'web application', can be used to execute more extensive, detailed evaluations and to compare design variations.

As part of a holistic view on the design, a 'daylight distribution map' may be generated illustrating the percentage of occupancy hours that an adjustable illumination threshold is achieved. Other aspects like running cost, life cycle cost or level of sustainability contributing to this approach are in prospect. 
As always, the designer, aware of simplifications and presumptions affecting the results, should do a critical review. The Sefaira software gives in that respect some guidance by putting the results in perspective with other concepts and adding rating scales.

The low-barrier program SketchUp is a preferred tool in conceptual design. It also gradually takes on features of BIM software, by providing tools to enrich the volumetric model with data or to use the conceptual model as input of BIM software. Hence we will briefly review the Open Studio program, which links SketchUp and EnergyPlus.

The designer starts configuring a model in the SketchUp interface. The Open Studio plug-in hereby adds a number of palettes to the toolbar. In SketchUp, one should decide in advance whether an architectural model or an energy model will be constructed, according to the vision that both require a different model view. In SketchUp, as opposed to BIM, it is not yet possible to derive an energy model from an architectural model. A specific feature of an energy model is that the user has to create volumes ('Spaces'), consisting of one or more rooms with the same thermal characteristics, rather than building elements such as walls and roofs. By selecting a particular element of the developed energy model, the designer can read out and modify the information available on that element. It concerns both data that the Open Studio plug-in automatically obtains through real-time analysis and data that one may add via the SketchUp interface. Auditing these values and being aware of their relative influence is crucial to interpret the analysis results correctly. The different render modes that are available assist herein by applying colour codes to the model, so one can visually verify whether all properties are correct.

The building energy model is loaded in the Open Studio application, directly from the SketchUp interface. The Open Studio application consists of a series of tabs, which one can step through from top to bottom to enter additional input or to inspect for modelling errors, in the run-up to the simulation. The user performs the simulation from within the interface of the Open Studio application, or imports the generated IDF file directly into EnergyPlus himself.

The strength of this Open Studio link is that it uses the well-renowned calculation engine EnergyPlus and that it starts from SketchUp, a program which is experienced as very convenient by most designers. However, it is unfortunate that one has to create a specific energy model instead of deriving it from an architectural model. Together with SketchUp, the Open Studio link loses its usefulness in the advanced design phases. In a rapidly evolving world of software the question is to which extent SketchUp will evolve to BIM software and whether this Open Studio link will continue to be successfully developed.

\subsection{Case Study 5: internal/concept stage - mass modelling and early energy evaluation within BIM software}

Quick-modelling CAD tools' main drawback is that the created Mass Model eventually needs to be introduced or rebuilt in BIM software. The lack of functionality for the conceptual design and the creation of free forms is in turn one of the disadvantages assigned to BIM applications. Some BIM software 
developers seized this lacuna as an opportunity to include the ability to create mass models within their software.

In Autodesk Revit, the 'mass modelling' tool emerged. This tool can not only be used to develop non-standard objects, but also to perform volume and shape studies in the conceptual design stage. A diverse range of forms and editing options provides the designer with the flexibility he needs to develop his ideas. Revit offers the possibility to carry out a first energy simulation on this conceptual model, on the condition that additional information is provided, ranging from building location and function to the level of insulation of the constructions and the targeted percentage glazing. Several alternatives can readily be explored by assuming other default values, without the need to remodel anything. Subsequently the mass model may be integrated within BIM through a set of Building Maker tools (fig. 3). These link Revit building elements to the surfaces of the mass model, the latter remaining present and forming the basis of a more extended BIM model. Even if one prefers an external CAD environment (such as SketchUp or AutoCAD 3D) the as such created mass model may be loaded in Revit at the desired instant. According to Autodesk, building a BIM model from scratch belongs to the past.

More recently, Graphisoft provided its BIM software ArchiCAD with a tool for freeform modelling, the 'morph' tool. At the points which have been examined in the context of this research - the possibility to perform energy simulations, to extract data relevant to an energy analysis and to administer the continuity of the design - this application appears to be less potent than the one in Revit. In ArchiCAD it is not possible to convert the faces of an existing morph into building model components. A conceptual scheme created with the morph tool or external CAD software cannot be leveraged, it provides no more than a support when creating a BIM model. Furthermore, a morph does not contain enough information to endorse an assessment by the Energy Evaluation tool. Nevertheless, in the foreseeable future ArchiCAD's morph tool shall probably turn out to be equally advanced as the one in Revit.
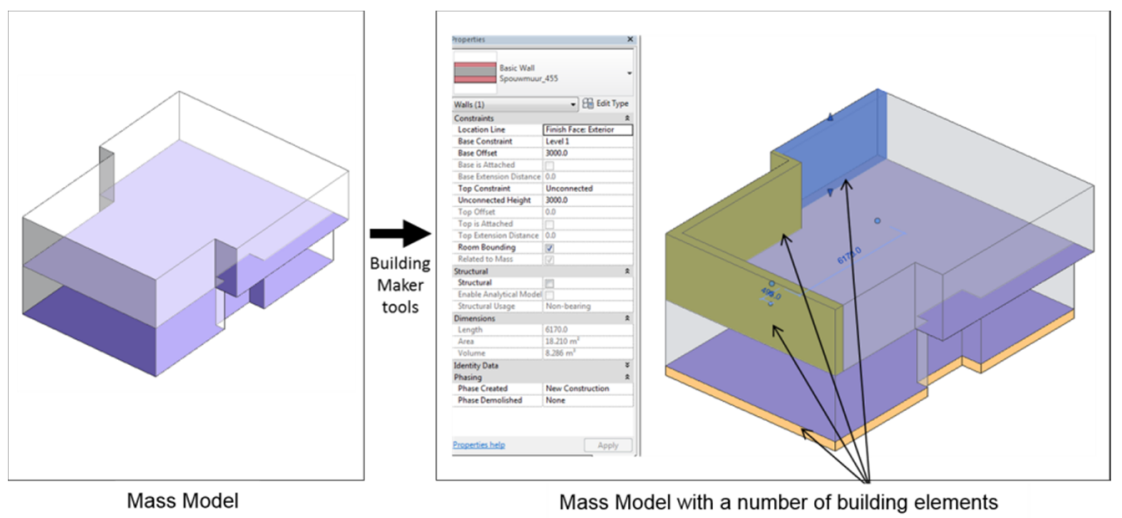

Figure 3: Operating the Building Maker tools, a volumetric model can be integrated within BIM. 


\section{Conclusion}

We may well conclude that a whole arsenal of tools exists to export data from a BIM model in favour of an energy simulation model. Yet automating the transfer to an application such as the Belgian EPB program is cumbersome, timeconsuming and may give rise to errors and misinterpretations. The development of tools to accomplish dynamic simulations by specialised software like EnergyPlus through the IFC standard is still in an experimental stage but offers good prospects for the operation of extensive data exchange and analysis. Likewise we see several promising developments on the market, both from BIM applications and energy simulation tools.

\section{References}

[1] Eastman, C., Liston, K., Sacks, R. \& Teicholz, P., BIM Handbook: A Guide To Building Information Modelling for Owners, Managers, Designers, Engineers and Contractors (2nd ed.), Wiley: Hoboken, 2011.

[2] Bernstein, H.M. \& Jones, S.A. (eds.), SmartMarket Report: The Business Value of BIM in North America. McGraw-Hill Construction: Bedford, 2012.

[3] ArchVision, Awareness and usage of BIM among architects is growing. British and Dutch architects have the lead, http://www.archvision.eu/persberichten/Awareness \& usage BIM among_architects_gro wing.British \& Dutch architects have lead.pdf, 2014.

[4] Maile, T., Fischer, M. \& Bazjanac, V., Building Energy Performance Simulation Tools - a Life-Cycle and Interoperable Perspective. Center for Integrated Facility Engineering (CIFE) Working Paper 107, 2007.

[5] Bazjanac, V., Maile, T., Rose, C., O’Donnell, J., Mrazović, N., Morrissey, E. \& Welle, B., An Assessment of the Use of Building Energy Performance Simulation in Early Design. Proc. of the $12^{\text {th }}$ Conf. of the IBPSA, Sydney, Australia, pp. 1579-1585, 2011.

[6] Bazjanac, V. \& Kiviniemi, A., Reduction, Simplification, Translation and Interpretation in the Exchange of Model Data. Proc. of the CIB-W78 $24^{\text {th }}$ Int. Conf. on Bringing ITC Knowledge to Work, Maribor, Slovenia, ed. D. Rebolj, pp. 163-168, 2007.

[7] Vlaams Energieagentschap, Nota: Beschermd volume, verliesoppervlakten en andere oppervlakten in het kader van energieprestatieregelgeving, http://www2.vlaanderen.be/economie/energiesparen/epb/doc/berekeningbv opp.pdf, 2014.

[8] Senave, M., Koppeling tussen BIM en energiesimulatie. [master dissertation], 2014.

[9] Space Boundary Tool (SBT), https://gaia.lbl.gov/interoperability/SBT/

[10] Maile, T., O’Donnell, J., Bazjanac, V. \& Rose, C., BIM - Geometry modelling guidelines for building energy performance simulation. Proc. of the $13^{\text {th }}$ Conference of IBPSA, Chambéry, France, pp. 3242-3249, 2013. 
[11] O’Donnell, J., Maile, T., Rose, C., Mrazović, N., Morrissey, E., Regnier, C., Parrish, K., \& Bazjanac, V., Transforming BIM to BEM: Generation of Building Geometry for the NASA Ames Sustainability Base BIM, LBNL Report 6033E, 2013.

[12] Bazjanac, V., IFC BIM-Based Methodology for Semi-Automated Building Energy Performance Simulation. Proc. of the CIB-W78 25 th Int. Conf. on Improving the Management of Construction Projects through IT Adoption, Santiago, Chile, ed. L. Rischmoller, C.C. Méndez: Chili, pp. 292-299, 2008.

[13] Bazjanac, V., Implementation of semi-automated energy performance simulation: building geometry. Proc. of the CIB-W78 $26^{\text {th }}$ Int. Conf. on Managing IT in Construction, Istanbul, Turkey, eds. A. Dikbas, E. Ergen \& H. Giritli, CRC Press/Balkema: Leiden, pp. 595-602, 2010.

[14] Simergy, http://simergy.lbl.gov/ 